\title{
Neurons and fractals: how reliable and useful are calculations of fractal dimensions?
}

\author{
Herbert F. Jelinek $^{\mathrm{a}, *}$, Eduardo Fernandez ${ }^{\mathrm{b}}$ \\ ${ }^{a}$ School of Community Health, Charles Sturt University, P.O. Box 789, Albury, NSW 2640, Australia \\ ${ }^{\mathrm{b}}$ Instituto de Bioingeniera, Universidad Miguel Hernandez, Elche, Spain
}

Received 8 August 1997; received in revised form 5 December 1997; accepted 7 December 1997

\begin{abstract}
In the past 15 years it has become possible to determine the fractal dimension (Df) of complex objects, including neurons, by automated image analysis methods. However, there are many unresolved issues that need to be addressed. In this paper we discuss how the Df calculated by different methods may vary and how fractal analysis may be of use for retinal ganglion cell characterization. The goal of this work was to acknowledge inherent sources of variation during measurement and evaluate current fractal analysis methods for describing structure. Our results show that different algorithms and even the same algorithm performed by different computer programs and/or experimenters may give different but consistent numerical values. All described methods demonstrated their suitability for classifying cat retinal ganglion cells into distinct groups. Our results reinforce the idea that comparison of measurements of different profiles using the same measurement method may be useful and valid even if an exact numeric value of the dimension is not realised in practice. (C) 1998 Elsevier Science B.V. All rights reserved.
\end{abstract}

Keywords: Fractal dimension; Analysis methods; Cat; Retinal ganglion cells

\section{Introduction}

Since Mandelbrot (1983) established fractal geometry to describe the complexity of forms found in nature, many analytical methods for determining the fractal dimension (Df) of natural objects have been published. All methods rely on the relationship between a measuring device and the spatial distribution of the data as points in space. Since not all methods give identical results for the same representation of an object, it has become important to establish guidelines for selecting a particular method and how these methods compare in order to standardise computation of Df.

This paper presents an analysis of different methods used to calculate Df of cat retinal ganglion cells. Each

\footnotetext{
* Corresponding author. Tel.: + 612 60516946; fax: + 612 60516898.
}

method presents some difficulties in its application and interpretation such that the various 'dimensions' that are measured by using the different methods are only equivalent for ideal (theoretical) fractal forms (Russ, 1994). To study how these different methods compare, we have analysed a set of 192 neuron images.

\section{Fractal geometry and naturally-occurring fractals}

Fig. 1 shows an example of an ideal/theoretical fractal object, the Koch curve, with a calculated Df of 1.26. The form of this object is complex since any change in magnification/scale will not alter the observed detail. This similarity observed between magnification/iteration levels is a feature of a geometrical object without a characteristic length and is termed self-similarity (Peitgen et al., 1992). 


\section{The fractal dimensions}

The Euclidean dimension describes objects in space as integers. Thus a straight line has a dimension of one $\left(D_{\mathrm{E}}=1\right)$, a plane a dimension of two $\left(D_{\mathrm{E}}=2\right)$ and a volume a dimension of three $\left(D_{\mathrm{E}}=3\right)$. Df, as a dimension, is simply a number that reflects a particular aspect of a geometric form. The dimension value is called fractal because it is a fraction and not a whole number. It is called dimension because it provides a measure of how completely an object fills space. Since cat retinal ganglion cells seen in two-dimensions are not straight lines and they do not completely cover the two-dimensional area, they cannot be adequately characterised by Euclidean geometry.

Obtaining a Df value for biological images by using the fractal analysis methods discussed below does not necessarily imply that the image or the representation of the image is fractal (Murray, 1995; Panico and Sterling, 1995). Natural objects are scale-invariant rather than self-similar within an upper and lower limit

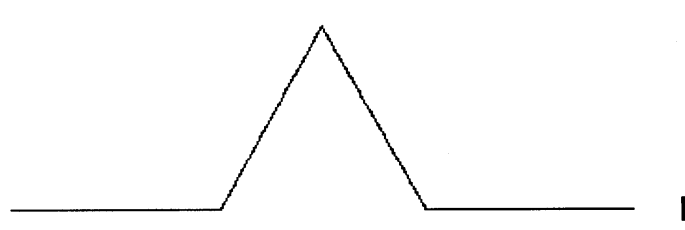

A
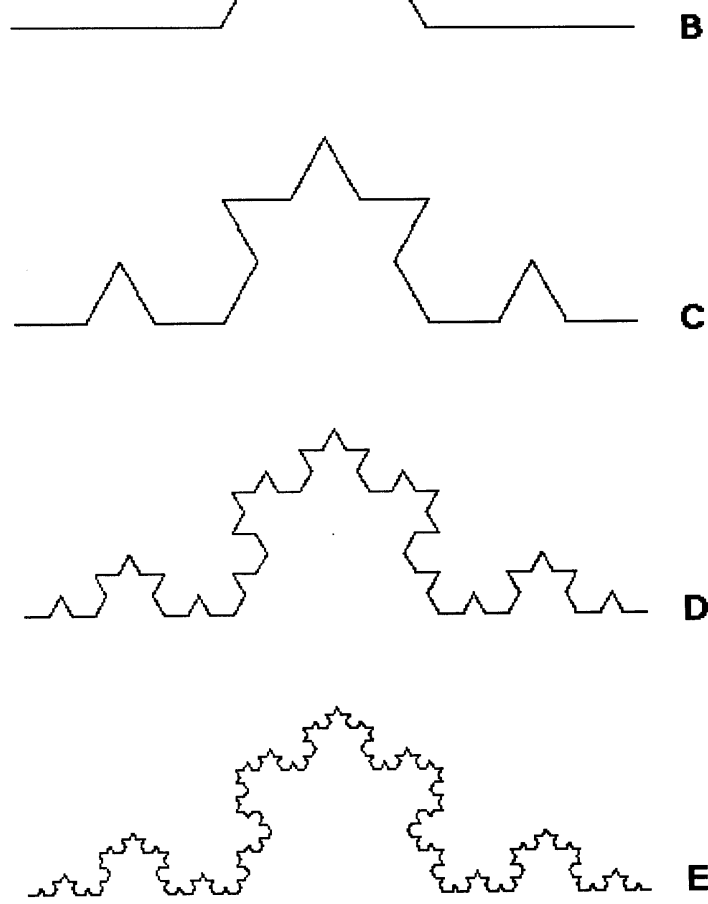

Fig. 1. The iterative construction of the Koch curve with a Df of 1.26. The sequential construction of this fractal begins with a straight line (A). Then, the middle third is raised to produce an equilateral triangle (B). Raising equilateral triangles from the middle third of each of the line segments in the object produces the image in (C). At higher stages of construction (D, E and so on) the fine detail of the complex curve would be lost due to resolution limits of the printing process. due to slight differences in detail between iteration levels (Peitgen and Richter, 1986). As the limited resolution of the screen does not allow a complete representation of any fractal object, a prefractal image is obtained and Df is estimated from this representation (Feder, 1998). The screen resolution also influences the border roughness of an object and leads to a deviation from the theoretical Df as well as from the expected linearity of the $\log -\log$ data points. Therefore, Df is not a very precise or accurate number and significance should not be extended beyond the second decimal place. However, Df is a useful additional parameter for object classification based on the 'complexity' of the object (Jelinek and Spence, 1997, 1998). The more complex the object or image, the more space filling it is and the fractional part of the Df value will be higher. A difference of 0.1 representing a doubling of complexity (Smith et al., 1989).

A number of different Dfs exist, many of which receive considerable attention in the mathematics literature (Falconer, 1985; Takayasu, 1990; Cross, 1994). We will only consider the Hausdorff and the MinkowskiBouligand dimension, as these are potentially useful for quantitative morphology. Algorithms based on these dimensions include the calliper, box-counting, mass-radius, dilation and cumulative intersection methods (Falconer, 1985; Peitgen and Richter, 1986; Schierwagen, 1990; Takayasu, 1990; Nonnenmacher et al., 1994; Jelinek et al., 1996). The calliper, box counting and dilation methods are all measures of length, while the mass-radius and cumulative intersection methods measure mass.

\section{Methods}

Data was obtained from 192 previously published images of retinal neurons that were either stained and classified solely on their morphology or stained after physiological recordings (Boycott and Wässle, 1974; Stone and Clarke, 1980; Wässle et al., 1981a,b; Saito, 1983; Stanford and Sherman, 1984; Stanford, 1987a,b; Wässle et al., 1987; Ramoa et al., 1988; Wässle and Boycott, 1991; Hutsler et al., 1993; Kolb et al., 1994; Pu et al., 1994). In addition, unpublished cells from wholemount retinae, provided by Jonathan Stone, (University of Sydney) were included in the analysis. All cells were drawn using camera lucida projection, as seen in wholemount preparations.

Since there is some divergence in the literature as to the 'correct' way an object is displayed for applying fractal analysis (Rigaut, 1983; Smith et al., 1996) all the images were analyzed in three ways: as binary or real drawings (black-on-white), as one-pixel-wide border images and as skeletonised tracings (Fig. 2). All transfor- 

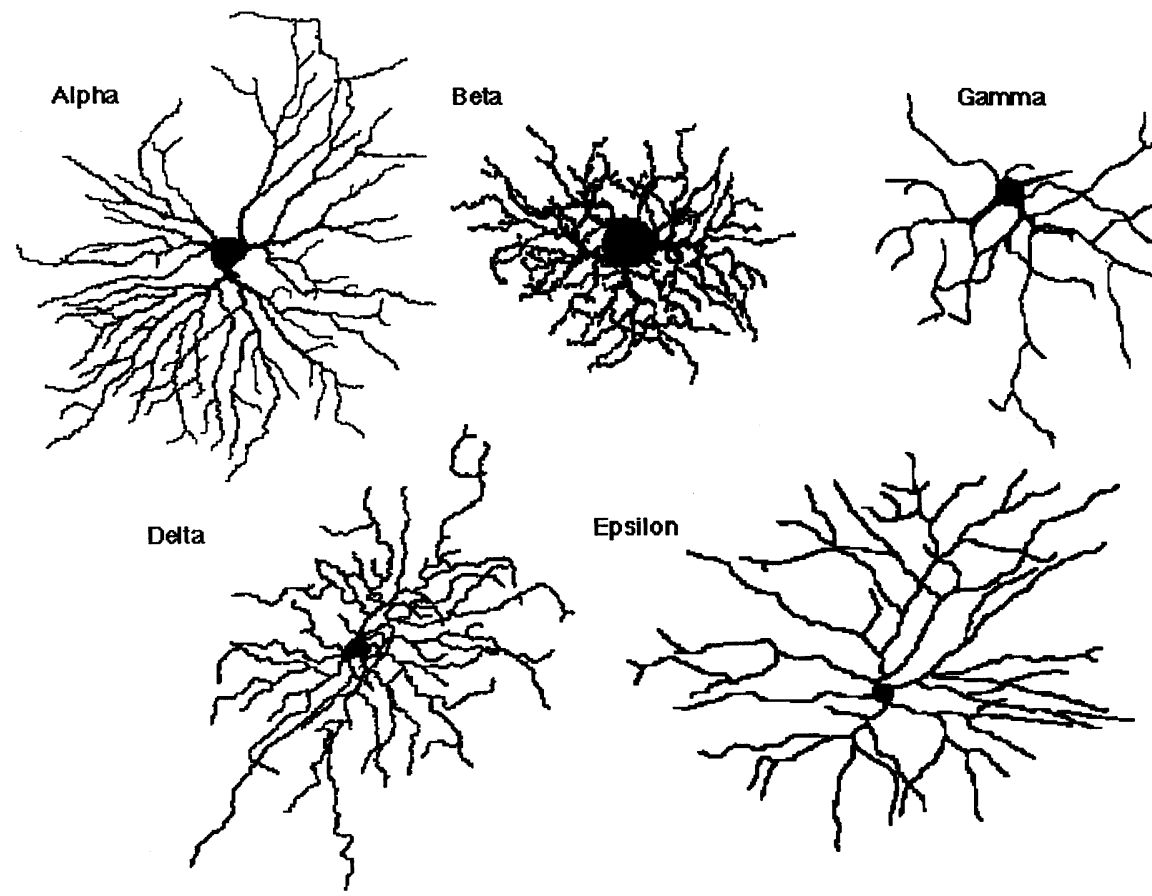

Fig. 4. Wholemount view of five different ganglion cell classes in cat retina.

possible choices of local origin are averaged and the average cluster mass $M(r)$ is obtained. The double logarithmic plot of $M(r)$ against $r$ gives a quantitative value for Df (Landini and Rippin, 1993; Caserta et al., 1995).

While both methods use 'boxes', they do so in a fundamentally different way. In box counting, the boxes are parts of grids which are laid over the image and the number of non-empty boxes are counted. The $\log$ ( \# boxes $\times$ box edge size) versus log (box edge size) plots give the capacity dimension. It is analogous to the calliper method in that it is an equivalent perimeter and equivalent ruler. It leads to the $D(0)$ dimension or zeroth moment of the $D(q)$ 's in multifractal analysis. With the sandbox method, the boxes are randomly centered on the fractal and the number of pixels are counted. Here the log counts or log mean count is plotted against the log box size. Thus it is a measure of mass or density with a different $D(q)$ (Smith et al., 1996).

The dilation method is based on the MinkowskiBouligand dimension, (Mandelbrot, 1983; Schroeder, 1991). A common form of this algorithm, as devised by Flook (1978), has been implemented by Smith et al. (1989) and others (Amthor, 1988; Porter et al., 1991; Smith et al., 1991; Siegel et al., 1991; Wingate et al., 1992; Neale et al., 1993; Smith et al., 1993; Smith and Behar, 1994). The dilation method replaced each pixel of the border by a circle whose diameter ranged from 3 to 61 pixels. Applying a convolution procedure (see NIH macros) structures smaller than the current diame- ter of the circle were filtered out. The length of the border for each respective diameter was then determined by the area of the outline divided by the diameter and Df estimated from the slope of the $\log -\log$ plot of length against diameter (Fig. 3B).

The cumulative intersection method was developed by Schierwagen (1990) and based on the method described by Stell and Witkovsky (1973). The Df was defined as $\mathrm{Df}=\log N c(r) / \log (r)$ where $N c(r)$ equalled the number of cumulative intersections at each concentric circle with increasing radii and $r$ the circle number from the centre. Fractop is an extension of this method. The analysis is performed and averaged over a number of centres (determined by user) that all lie within a ratio of the radius of gyration (determined by user). For each of the centre points, the analysis function is then called up and returns an array of the cumulative sums of the intersections versus radius from the centre point (Fig. 3D). The gradient of the log of the cumulative intersections versus $\log$ of the radius is then directly related to the Df (Jelinek et al., 1996).

Before beginning with the analysis of retinal neurons, every method was tested against a variety of fractals of known Df. The fractals that were used as testing images were either scanned from the book of Mandelbrot (1983) or generated using different routines written by the authors. In addition, we also analysed Euclidean objects such as lines, circles and areas. To avoid potential problems, such as anisotropy or preferred origin of the measurement method, 10 measures from each image were computed for different orientations and origins. 
The correct Df for each profile was considered as the average value of all the measurements.

Statistical differences were analysed using the SPSS/ $\mathrm{PC}+$ software package (SPSS). The Dfs of the same cell drawing using different methods and image transformations (binary silhouettes, one-pixel-wide borders or skeletonised images) were compared using the Scheffe $F$-test (Welkowitz et al., 1976).

We also investigated the importance of each measurement method in relationship to discriminating or classifying different morphological ganglion cell classes. Fig. 4 shows a wholemount view of the five different ganglion cell types that we have considered in this study. We used the discriminant analysis module of the SPSS/PC + based on Baye's rule. This procedure al-

Table 1

Fractal analysis of objects of known fractal dimension

\begin{tabular}{|c|c|c|c|}
\hline Figure & Box counting & Dilation & Mass-radius \\
\hline \multicolumn{4}{|l|}{ Line $(\mathrm{D}=1)$} \\
\hline Silhouettes & $0.99 \pm 0.01$ & $1.00 \pm 0.01$ & $0.99 \pm 0.01$ \\
\hline Outlines & $0.99 \pm 0.01$ & $1.00 \pm 0.01$ & $0.99 \pm 0.01$ \\
\hline Skeleton & $0.99 \pm 0.01$ & $1.00 \pm 0.01$ & $0.99 \pm 0.01$ \\
\hline \multicolumn{4}{|l|}{ Circle $(\mathrm{D}=1)$} \\
\hline Silhouettes & $1.01 \pm 0.01$ & $1.00 \pm 0.01$ & $0.99 \pm 0.01$ \\
\hline Outlines & $1.01 \pm 0.01$ & $1.00 \pm 0.01$ & $0.99 \pm 0.01$ \\
\hline Skeleton & $1.05 \pm 0.01$ & $0.98 \pm 0.01$ & $0.99 \pm 0.01$ \\
\hline \multicolumn{4}{|c|}{ Koch coastline $(\mathrm{D}=1.26)$} \\
\hline Silhouettes & $1.21 \pm 0.01$ & $1.24 \pm 0.01$ & $1.25 \pm 0.01$ \\
\hline Outlines & $1.21 \pm 0.01$ & $1.24 \pm 0.01$ & $1.25 \pm 0.01$ \\
\hline Skeleton & $1.15 \pm 0.01$ & $1.23 \pm 0.01$ & $1.24 \pm 0.01$ \\
\hline \multicolumn{4}{|c|}{ Koch Island $(\mathrm{D}=1.50)$} \\
\hline Silhouettes & $1.48 \pm 0.02$ & $1.52 \pm 0.01$ & $1.63 \pm 0.02$ \\
\hline Outlines & $1.46 \pm 0.01$ & $1.50 \pm 0.01$ & $1.45 \pm 0.02$ \\
\hline Skeleton & $1.37 \pm 0.01$ & $1.40 \pm 0.01$ & $1.54 \pm 0.01$ \\
\hline \multicolumn{4}{|c|}{ Mandelbrot curve $(\mathrm{D}=1.63)$} \\
\hline Silhouettes & $1.55 \pm 0.01$ & $1.57 \pm 0.01$ & $1.57 \pm 0.01$ \\
\hline Outlines & $1.55 \pm 0.01$ & $1.58 \pm 0.01$ & $1.63 \pm 0.01$ \\
\hline Skeleton & $1.50 \pm 0.01$ & $1.54 \pm 0.01$ & $1.58 \pm 0.01$ \\
\hline \multicolumn{4}{|c|}{ Cluster $(\mathrm{D}=1.71)$} \\
\hline Silhouettes & $1.65 \pm 0.01$ & $1.65 \pm 0.01$ & $1.76 \pm 0.01$ \\
\hline Outlines & $1.65 \pm 0.01$ & $1.66 \pm 0.01$ & $1.74 \pm 0.01$ \\
\hline Skeleton & $1.61 \pm 0.01$ & $1.63 \pm 0.01$ & $1.58 \pm 0.02$ \\
\hline \multicolumn{4}{|c|}{ Koch box $(\mathrm{D}=1.80)$} \\
\hline Silhouettes & $1.82 \pm 0.01$ & $1.87 \pm 0.01$ & $1.90 \pm 0.01$ \\
\hline Outlines & $1.82 \pm 0.01$ & $1.87 \pm 0.01$ & $1.90 \pm 0.01$ \\
\hline Skeleton & $1.82 \pm 0.01$ & $1.87 \pm 0.01$ & $1.87 \pm 0.01$ \\
\hline \multicolumn{4}{|c|}{ Triangle $(\mathrm{D}=1.94)$} \\
\hline Silhouettes & $1.72 \pm 0.01$ & $1.80 \pm 0.01$ & $1.74 \pm 0.01$ \\
\hline Outlines & $1.66 \pm 0.01$ & $1.80 \pm 0.01$ & $1.74 \pm 0.01$ \\
\hline Skeleton & $1.77 \pm 0.02$ & $1.78 \pm 0.01$ & $1.72 \pm 0.01$ \\
\hline \multicolumn{4}{|l|}{ Square $(D=2)$} \\
\hline Silhouettes & $1.99 \pm 0.01$ & $1.90 \pm 0.01$ & $1.93 \pm 0.01$ \\
\hline Outlines & $1.02 \pm 0.01$ & $1.00 \pm 0.01$ & $1.00 \pm 0.01$ \\
\hline Skeleton & $0.06 \pm 0.01$ & $0.23 \pm 0.01$ & $0.12 \pm 0.02$ \\
\hline
\end{tabular}

Table 2

Mean fractal values using different methods

\begin{tabular}{|c|c|c|c|c|c|}
\hline Method & Mean & S.D. & Range & Minimum & $\begin{array}{l}\text { Maxi- } \\
\text { mum }\end{array}$ \\
\hline \multicolumn{6}{|c|}{ Box counting 1 (Image 1.58) } \\
\hline Silhouettes & 1.42 & 0.13 & 0.62 & 1.09 & 1.71 \\
\hline Outlines & 1.37 & 0.12 & 0.58 & 1.09 & 1.67 \\
\hline Skeleton & 1.22 & 0.15 & 0.69 & 1.00 & 1.67 \\
\hline \multicolumn{6}{|c|}{ Box counting 2 (Image Fractal 1.2) } \\
\hline Silhouettes & 1.41 & 0.11 & 0.56 & 1.09 & 1.65 \\
\hline Outlines & 1.37 & 0.11 & 0.67 & 1.08 & 1.75 \\
\hline Skeleton & 1.24 & 0.13 & 1.14 & 0.32 & 1.46 \\
\hline \multicolumn{6}{|c|}{ Box counting 3 (University of Otago) } \\
\hline Silhouettes & 1.39 & 0,11 & 0.52 & 1.09 & 1.60 \\
\hline Outlines & 1.34 & 0.10 & 0.45 & 1.06 & 1.51 \\
\hline Skeleton & 1.20 & 0.09 & 0.50 & 0.95 & 1.45 \\
\hline \multicolumn{6}{|c|}{ Grid intercept method (Harvard) } \\
\hline Silhouettes & 1.37 & 0.17 & 0.79 & 1.05 & 1.85 \\
\hline Outlines & 1.24 & 0.08 & 0.43 & 0.97 & 1.41 \\
\hline Skeleton & 1.19 & 0.09 & 0.42 & 0.97 & 1.40 \\
\hline \multicolumn{6}{|c|}{ Dilation method (NIH) } \\
\hline Silhouettes & 1.41 & 0.13 & 0.69 & 1.04 & 1.73 \\
\hline Outlines & 1.35 & 0.10 & 0.50 & 1.04 & 1.54 \\
\hline Skeleton & 1.30 & 0.10 & 0.52 & 1.02 & 1.54 \\
\hline \multicolumn{6}{|c|}{ Mass fractal (NIH) } \\
\hline Silhouettes & 1.48 & 0.15 & 0.69 & 1.10 & 1.79 \\
\hline Outlines & 1.40 & 0.11 & 0.64 & 1.07 & 1.71 \\
\hline Skeleton & 1.28 & 0.12 & 0.63 & 1.01 & 1.64 \\
\hline \multicolumn{6}{|c|}{ Mass fractal (Charles Sturt University) } \\
\hline Silhouettes & 1.43 & 0.17 & 0.80 & 0.95 & 1.74 \\
\hline Outlines & 1.55 & 0.28 & 3.61 & 1.03 & 4.64 \\
\hline Skeleton & 1.43 & 0.15 & 0.78 & 0.99 & 1.77 \\
\hline \multicolumn{6}{|c|}{ Cumulative intersection method (Charles Sturt University) } \\
\hline Silhouettes & 1.67 & 0.27 & 1.58 & 0.99 & 2.57 \\
\hline Outlines & 1.54 & 0.17 & 0.89 & 0.99 & 1.87 \\
\hline Skeleton & 1.44 & 0.14 & 0.70 & 1.02 & 1.72 \\
\hline
\end{tabular}

lows classification of a cell into the appropriate cell group and provides detailed information on the results of the classification phase (Fernandez et al., 1994).

\section{Results}

\subsection{Analysis of images with known Df}

The estimated Df of standard objects obtained with all methods was not significantly different to the calculated/nominal value. The results of the most often used methods - the box counting, dilation and mass-radius analysis - are shown in Table 1. Different spatial orientations and/or origin of the box mesh yielded slightly different results, introducing some minor variations in the numerical values of Df, probably due to the anisotropy of the profiles (Jelinek, 1996). All methods showed similar trends in the results, differing in some 
Table 3

Mean and S.D. $(M \pm$ S.D. $)$ of fractal dimension in different classes of cat ganglion cells

\begin{tabular}{|c|c|c|c|c|c|}
\hline \multirow[t]{2}{*}{ Fractal method } & \multicolumn{5}{|l|}{ Cell class } \\
\hline & $\alpha$ & $\beta$ & $\gamma$ & $\delta$ & $\epsilon$ \\
\hline \multicolumn{6}{|c|}{ Box counting (Image 1.58) } \\
\hline Silhouettes & $1.45 \pm 0.07$ & $1.55 \pm 0.06$ & $1.27 \pm 0.08$ & $1.43 \pm 0.03$ & $1.37 \pm 0.05$ \\
\hline Outlines & $1.44 \pm 0.06$ & $1.49 \pm 0.09$ & $1.25 \pm 0.07$ & $1.43 \pm 0.03$ & $1.35 \pm 0.03$ \\
\hline Skeleton & $1.31 \pm 0.08$ & $1.34 \pm 0.14$ & $1.02 \pm 0.26$ & $1.30 \pm 0.07$ & $1.25 \pm 0.07$ \\
\hline \multicolumn{6}{|c|}{ Box counting (Image Fractal 1.2) } \\
\hline Silhouettes & $1.45 \pm 0.05$ & $1.49 \pm 0.07$ & $1.25 \pm 0.06$ & $1.44 \pm 0.02$ & $1.37 \pm 0.05$ \\
\hline Outlines & $1.44 \pm 0.04$ & $1.44 \pm 0.08$ & $1.23 \pm 0.05$ & $1.43 \pm 0.02$ & $1.35 \pm 0.04$ \\
\hline Skeleton $\int$ & $1.35 \pm 0.05$ & $1.31 \pm 0.05$ & $1.09 \pm 0.06$ & $1.33 \pm 0.04$ & $1.19 \pm 0.25$ \\
\hline \multicolumn{6}{|c|}{ Box counting (University of Otago) } \\
\hline Silhouettes & $1.43 \pm 0.05$ & $1.48 \pm 0.06$ & $1.26 \pm 0.05$ & $1.41 \pm 0.03$ & $1.33 \pm 0.05$ \\
\hline Outlines & $1.39 \pm 0.07$ & $1.40 \pm 0.07$ & $1.23 \pm 0.04$ & $1.40 \pm 0.03$ & $1.31 \pm 0.03$ \\
\hline Skeleton & $1.28 \pm 0.03$ & $1.26 \pm 0.03$ & $1.06 \pm 0.06$ & $1.27 \pm 0.04$ & $1.20 \pm 0.04$ \\
\hline \multicolumn{6}{|c|}{ Grid intercept method (Harvard) } \\
\hline Silhouettes & $1.32 \pm 0.08$ & $1.49 \pm 0.07$ & $1.35 \pm 0.10$ & $1.29 \pm 0.07$ & $1.26 \pm 0.09$ \\
\hline Outlines & $1.26 \pm 0.05$ & $1.33 \pm 0.05$ & $1.24 \pm 0.04$ & $1.25 \pm 0.04$ & $1.22 \pm 0.05$ \\
\hline Skeleton $\int$ & $1.25 \pm 0.05$ & $1.32 \pm 0.05$ & $1.22 \pm 0.04$ & $1.22 \pm 0.04$ & $1.19 \pm 0.04$ \\
\hline \multicolumn{6}{|l|}{ Dilation (NIH) } \\
\hline Silhouettes & $1.44 \pm 0.06$ & $1.53 \pm 0.06$ & $1.27 \pm 0.06$ & $1.43 \pm 0.02$ & $1.36 \pm 0.05$ \\
\hline Outlines & $1.41 \pm 0.06$ & $1.46 \pm 0.06$ & $1.22 \pm 0.05$ & $1.22 \pm 0.05$ & $1.34 \pm 0.05$ \\
\hline Skeleton $\int$ & $1.37 \pm 0.05$ & $1.42 \pm 0.05$ & $1.20 \pm 0.05$ & $1.22 \pm 0.05$ & $1.27 \pm 0.06$ \\
\hline \multicolumn{6}{|c|}{ Mass fractal (NIH) } \\
\hline Silhouettes & $1.49 \pm 0.06$ & $1.62 \pm 0.08$ & $1.31 \pm 0.11$ & $1.46 \pm 0.04$ & $1.46 \pm 0.07$ \\
\hline Outlines & $1.43 \pm 0.05$ & $1.51 \pm 0.10$ & $1.27 \pm 0.09$ & $1.46 \pm 0.04$ & $1.40 \pm 0.05$ \\
\hline Skeleton $\int$ & $1.36 \pm 0.07$ & $1.38 \pm 0.13$ & $1.13 \pm 0.09$ & $1.36 \pm 0.05$ & $1.33 \pm 0.10$ \\
\hline \multicolumn{6}{|c|}{ Mass fractal (Charles Sturt University) } \\
\hline Silhouettes & $1.51 \pm 0.11$ & $1.53 \pm 0.10$ & $1.28 \pm 0.16$ & $1.53 \pm 0.11$ & $1.45 \pm 0.15$ \\
\hline Outlines & $1.62 \pm 0.07$ & $1.72 \pm 0.10$ & $1.38 \pm 0.11$ & $1.60 \pm 0.07$ & $1.68 \pm 0.08$ \\
\hline Skeleton $\int$ & $1.59 \pm 0.07$ & $1.60 \pm 0.08$ & $1.32 \pm 0.12$ & $1.54 \pm 0.11$ & $1.47 \pm 0.13$ \\
\hline \multicolumn{6}{|c|}{ Cumulative intersection method (Charles Sturt University) } \\
\hline Silhouettes & $1.75 \pm 0.10$ & $2.04 \pm 0.22$ & $1.45 \pm 0.13$ & $1.72 \pm 0.25$ & $1.57 \pm 0.13$ \\
\hline Outlines & $1.66 \pm 0.07$ & $1.75 \pm 0.08$ & $1.39 \pm 0.09$ & $1.61 \pm 0.08$ & $1.50 \pm 0.09$ \\
\hline Skeleton & $1.64 \pm 0.06$ & $1.63 \pm 0.09$ & $1.31 \pm 0.12$ & $1.57 \pm 0.11$ & $1.49 \pm 0.13$ \\
\hline
\end{tabular}

minor yet consistent ways within and between laboratories.

An analysis of binary versus outlined or skeletonised images showed that binary images had higher fractal values than boundary or skeletonised images, although no statistically significant differences were observed. We should emphasise that we did not employ solid test figures (except for areas with a $\mathrm{Df}=2$ ) since these solid figures could not be reliably calibrated (for instance solid triadic or quadric Koch islands did not yield the expected Df values of 1.26 and 1.50).

\subsection{Classification of retinal ganglion cells by using different fractal analysis methods}

The mean Df values for groups of retinal neurons using different methods are shown in Table 2. We used an analysis of variance (ANOVA) for repeated measures and Scheffe Post Hoc test for matched pairs to investigate differences between means. There were significant differences in the mean Dfs $(p<0.0001)$ of individual groups, indicating that the numerical value of Df depended strongly on the method used to compute it. Even methods that in theory measured the same type of dimension (e.g. the different box counting) showed statistical differences in their measured Df values $(p<0.001)$ for identical cell groups. Df values were constant across all methods.

A strong correlation $(p<0.001)$ between all methods was observed. The only exceptions being the grid intersection and the cumulative mass-radius method, that showed a weaker correlation $(p<0.01)$. These results confirm the usefulness of fractal analysis as an objective and quantitative parameter of neural complexity. 


\subsection{Comparison of the same method by different experimenters}

In order to address whether the same method and computer implementation program gave an identical Df value, the same 192 neuron images were analysed by two different experimenters using the same computer programs, but different computers at different locations. Although there were slightly different Df values, these were not statistically significant. Furthermore all procedures were reproducible, with high correlation coefficients ranging from 0.964 to 1.0 .

\subsection{Comparison of binary, outlined and skeletonised images}

Binary images, independent of the fractal method, showed significantly higher fractal values $(p=0.001)$ compared with outlined and skeletonised. This could be because the cell interior, with a $\mathrm{Df}=2$, increases the Df value for binary images (Smith and Lange, 1995; Viscek, 1995).

\subsection{Discriminant analysis}

As we were interested in the anatomical and functional diversity of cat retinal ganglion cells, we studied the usefulness of Df as a quantitative measure. In the cat there are three morphologically distinct ganglion cell classes/types, the $\alpha, \beta$ and $\gamma$ that have been agreed on (Boycott and Wässle, 1974; Kolb et al., 1994). The $\gamma$ cell class contains several cell types including: the $\gamma$ cells (Boycott and Wässle, 1974; Kolb et al., 1994), the $\delta$ cells (Wässle et al., 1987; Dacey, 1989) and the $\varepsilon$ cells (Leventhal et al., 1985).

We posed the following questions: (1) Can fractal analysis be a useful tool to discriminate between these ganglion cell classes/types; and (2) which is the best way to determine Df in order to compare different morphological cat retinal ganglion cells? An ANOVA and a discriminant analysis to address these questions were used.

Although the range of Dfs for the $\alpha, \beta, \gamma, \delta$ and $\varepsilon$ cells overlapped (Table 3), an ANOVA showed that there were significant differences in their mean Df. A discriminant analysis using each method applied individually to binary, skeletonised and border only images, ranked these methods with the dilation method and the cumulative intersection procedure having the highest ranked score. Furthermore, classification, in agreement with the classification scheme previously suggested by Boycott and Wässle (1974), improved significantly when binary, instead of border only or skeletonised images were used. Table 4 shows the percentage of retinal cells correctly classified using different methods to compute the Df. Thus the dilation and cumulative intersection methods, when performed on binary images, classified a cell correctly with a respective accuracy of 65.3 and $62 \%$, in agreement with the Boycott and Wässle (1974). The discriminant analysis also showed that Df was a useful parameter for identifying different classes of retinal neurons $(p<0.001)$.

\section{Discussion}

The use of different fractal analysis methods in biology is not solely based on whether an object is scale-invariant and therefore fractal. It can be used as a robust tool allowing differentiation of morphological features, such as the complexity of the dendritic branching pattern that are difficult to objectively evaluate using alternative analytical methods.

No significant differences were obtained using one particular method, although manipulation of image

Table 4

Percentage of cat ganglion cells classes correctly classified using only their fractal dimension values

\begin{tabular}{|c|c|}
\hline Fractal method & $(\%)$ \\
\hline \multicolumn{2}{|c|}{ Box counting $(\mathrm{NIH})$} \\
\hline Silhouettes & 59.2 \\
\hline Outlines & 55.1 \\
\hline Skeleton & 43.5 \\
\hline \multicolumn{2}{|c|}{ Box counting (Image Fractal 1.2) } \\
\hline Silhouettes & 53.1 \\
\hline Outlines & 52.2 \\
\hline Skeleton & 46.1 \\
\hline \multicolumn{2}{|c|}{ Box counting (University of Otago) } \\
\hline Silhouettes & 47.9 \\
\hline Outlines & 54.3 \\
\hline Skeleton & 50.0 \\
\hline \multicolumn{2}{|c|}{ Grid intercept method } \\
\hline Silhouettes & 41.2 \\
\hline Outlines & 38.1 \\
\hline Skeleton & 35.4 \\
\hline \multicolumn{2}{|l|}{ Dilation } \\
\hline Silhouettes & 65.3 \\
\hline Outlines & 57.1 \\
\hline Skeleton & 45.9 \\
\hline \multicolumn{2}{|c|}{ Mass fractal (from NIH) } \\
\hline Silhouettes & 59.8 \\
\hline Outlines & 46.7 \\
\hline Skeleton & 39.1 \\
\hline \multicolumn{2}{|l|}{ Mass fractal } \\
\hline Silhouettes & 36.7 \\
\hline Outlines & 44.6 \\
\hline Skeleton & 36.7 \\
\hline \multicolumn{2}{|c|}{ Cumulative intersection method } \\
\hline Silhouettes & 61.9 \\
\hline Outlines & 53.3 \\
\hline Skeleton & 36.7 \\
\hline
\end{tabular}


content (e.g. binary, skeletonised) showed that skeletonised images had consistently lower Df values. The lower estimated Df values for skeletonised images are a result of removing one of the main contributions to image complexity, that is the border ruggedness and the space filling effect of the cell interior.

Since we did obtain different numerical values for Df using different methods and implementation programs for the same method, we wanted to ascertain if limitations or problems with the analysis methods could account for these differences. Possible problems with some methods included measurements with large measuring elements that exceed the bounds of the image frame, giving erroneous plots. To avoid this problem we made the image occupy only $2 / 3$ of the image frame. With the NIH Image version of the dilation method, large elements that exceeded the bounds of the image frame count zero and therefore do not contribute to the estimate of Df. Another problem, especially with the box counting method from New Zealand, is that in order to get an unbiased estimate of Df, it is necessary to find the minimum number of boxes of a particular size that cover the object. This can be achieved by offsetting the origin of the initial grid, but this is a very time consuming procedure, that usually did not report more than $0.5 \%$ decrease in error (Bourke, 1993). A more important problem is how the line of best fit is obtained from the data to determine the slope and hence Df. Since, for some methods, the points are not uniformly spaced and the regression fit should not necessarily weigh all the points equally, this is not an entirely trivial operation (Russ, 1994). However, for the box-counting methods discussed, the box sizes are scaled as a power of 2 and are therefore evenly spaced. Selecting only a portion of the data points, fitting more than one regression line to the data points and taking the average of several slopes along the data points, are all valid alternatives for estimating Df but may result in different values (Jelinek and Spence, 1998).

Although the difference between the estimated dimensions is usually small in comparison to observational error (Takayasu, 1990), different Dfs (measuring length or mass) return different information about the image. This means that different methods may quantify different aspects of the image and thus it is important to establish some criteria for choosing a particular method. Since the interpretation of the numeric values of Df, as though the data were from an ideal fractal should be avoided, our approach has been to evaluate the usefulness of the different methods to compute Df for classification tasks based on pattern description. Our results showed that all fractal analysis methods yielded consistent Df values and that fractal analysis is a useful and statistically significant parameter to classify retinal ganglion cells. For retinal ganglion cell classification, the dilation and the cumulative intersec- tion procedures performed on binary silhouettes provided the most successful correlation compared with accepted classification schemes.

It should be noted that Df is only a descriptive parameter, like the dendritic field area or the size of the soma and does not necessarily imply any underlying mechanism of form generation or function. In general, a connection between empirical values of Df and any specific growth mechanism should be avoided and require the answering of specific experimental questions. The question whether particular biological images are fractal has seen some controversy with Panico and Sterling (1995) and Murray (1995) suggesting that neuron and vascular patterns are space filling as their $\mathrm{Df}=2$ but not fractal. Retinal patterns showed no linear region in the $\log -\log$ plots, there was no effect of disarrangement on the $\log -\log$ plots and no difference to any other patterns of branched line segments (Panico and Sterling, 1995). The following comments address the three points raised by these authors. Unfortunately $\log -\log$ plots were not shown in the Panico and Sterling paper and therefore only comments pertaining to their semi-log results and general discussion can be made here. Montague and Friedlander (1989), who analysed the retinal neuron originally and Landini (1995) who analysed retinal blood vessels, observed linear $\log -\log$. It remains doubtful if the measurement of local slopes along the curve of the $\log -\log$ plot and plotting these as a bar graph over a six-point window and sliding the window along the entire $\log$-log curve is a good procedure.

Previous results, published by Caserta et al. (1995) and corroborated in our laboratory $(\mathrm{HJ})$, suggested that the linearity of the plot depends on the size of the window and that the size of that window influenced the magnitude of Df. A Df of 2 implies that the image covers the plane completely which is not the case when the images are viewed on screen (Fig. 4; Panico and Sterling, 1995) and may also be a consequence of the range of box sizes or circles chosen (Caserta, pers. comm.). Murray (1995) and Panico and Sterling (1995) concluded that images that are space filling are not fractal. Fractals, however, are defined by their space filling complexity. The greater the complexity of the image, the greater it's space filling capacity. Peitgen et al. (1992) pointed out that not all self-similar objects are fractals and cite the peano curve and the devil's staircase as examples. These two images are fractal objects as they display self-similarity (peano curve) or self-affine scaling (devil's staircase) but have dimension values of 2 and 1, respectively. Images such as retinal ganglion cells that have overlapping processes may also have dimension values $>2$. In addition, randomising a structure results in spreading the structure out evenly over the plane and will give, on average, a dimension of 2 and is not a good test for fractality. Thus, self-similar- 
ity or the Df value are not absolute indicators of whether an image is fractal.

Notwithstanding the above-mentioned limitations, it remains that in many situations a single number, the Df, summarises concisely the amount of detail and complexity of neurons (Fernandez et al., 1994). Furthermore, fractal geometry has some other advantages over its integer-dimensional counterparts. Shrinkage or expansion of a neuron will not affect their fractal values as long as the artefact acts equally in all directions and the measured points still lie on the linear segment of the graph. This means that the Dfs of different neurons that have been processed in different batches or at different laboratories can usually be compared directly (as long as the same methodology used to calculate Df is applied).

In summary, an approximation of the Df of natural objects is now possible by various methods. We suggest that Df values can be an objective and useful parameter to characterise the morphological complexity of neurons. Our results reinforce the idea that comparison of measurements of different profiles, using the same measurement method, may be useful and valid even if the exact numeric value of the dimension is not necessarily very accurate. Finally we propose that the Df value of a neuron should be used, in addition to the other morphometric parameters typically used to achieve a rigorous understanding of neural morphology.

\section{Acknowledgements}

This work was supported by a DGICYT Research grant PB94/1509 to E.F. Assistance with the image analysis by Peter Bowdren and Cherryl Kolbe is gratefully acknowledged. The code for the cumulative intersection method and mass-radius method in Fractop was written by Tony Steinke. Fractop can be found at http://csu.edu.au/fractop. Careful reading of the manuscript and suggestions by Cameron Jones is appreciated.

\section{References}

Amthor FR. Quantitative fractal analysis of dendritic trees of identified rabbit retinal ganglion cells. Soc Neurosci 1988;14(1):602.

Block A, von Bloh W, Schellnhuber HJ. Efficient box-counting determination of generalized fractal dimension. Phys Rev A 1990;42(4):1869-74.

Bourke P. Fractal dimension calculator manual. http:// www.mhri.edu.au/ pdb/fractals/fracdim.html, 1993.

Boycott BB, Wässle H. The morphological types of ganglion cells of the domestic cat's retina. J Physiol 1974;240:397-419.

Caserta F, Eldred WD, Fernandez E, Hausman RE, Stanford LR, Bulderev SV, Schwarzer S, Stanley HE. Determination of physiologically characterized neurons in two and three dimensions. J Neurosci Methods 1995;56:133-44.
Cross SS. The application of fractal geometric analysis to microscopic images. Micron 1994;25:101-13.

Dacey DM. Monoamine-acumulating ganglion cell type of the cat's retina. J Comp Neurol 1989;288:59-80.

Falconer KJ. The Geometry of Fractal Sets. Cambridge: Cambridge University Press, 1985.

Feder J. Fractals. New York: Plenum Press, 1998.

Fernandez E, Guiloff G, Kolb H, Ammermüller D, Zhang D, Eldred W. Fractal dimension as a useful parameter for morphological classification of retinal neurons. Invest Ophthalmol Vis Sci 1992;33:S940.

Fernandez E, Eldred WD, Ammermüller J, Block A, von Bloh W, Kolb H. Complexity and scaling properties of amacrine, ganglion, horizontal and bipolar cells in the turtle retina. J Comp Neurol 1994;347:397-408.

Flook AG. The use of dilation logic on the Quantimet to achieve fractal dimension characterisation of textured and structured profiles. Powder Techn 1978;21:295-8.

Honda E, Domon M, Sasaki T. A method for determination of fractal dimensions of sialographic images. Invest Radiol 1991;26:894-901.

Hutsler JJ, White CA, Chalupa LM. Neuropeptide Y immunoreactivity identifies a group of gamma-type retinal ganglion cells in the cat. J Comp Neurol 1993;336:468-80.

Jelinek HF. The use of fractal analysis in cat retinal ganglion cell classification. PhD Thesis, The University of Sydney, 1996.

Jelinek HF, Spence I. Categorisation of physiologically and morphologically characterised non- $\alpha /$ non- $\beta$ cat retinal ganglion cells using fractal geometry. Fractal 1997;5(4):673-84.

Jelinek HF, Spence I. Criteria for the use of fractal analysis in neuroscience. J Hirnforschung 1998; (submitted).

Jelinek HF, Cox G, Spence I. Fractal analysis of rat ventral horn neurones. Proc Aust Neurosci Soc 1992;3:121.

Jelinek HF, Bowdren P, Spence I. Form and function of cat retinal ganglion cells: the $\mathrm{X}$ and $\mathrm{Y}$ cells revisited using fractal analyis. Int J Neurosci 1996;89:99.

Kolb H, Fernandez E, Schouten J, Ahnelt P, Linberg KA, Fisher SK. Are there three types of horizontal cells in the human retina? $\mathrm{J}$ Comp Neurol 1994;343:370-86.

Landini G. Applications of fractal geometry in pathology. In: Iannaccone PM, Khokha M, editors. Fractal Geometry in Biological Sciences. Boca Raton, FL: CRC Press, 1995.

Landini G, Rippin JW. Notes on the implementation of the mass-radius method of fractal dimension estimation. Comput Appl Biosci 1993;9(5):547-50.

Leventhal A, Rodieck RW, Dreher B. Central projections of the cat retinal ganglion cells. J Comp Neurol 1985;237:216-26.

Mandelbrot BB. The Fractal Geometry of Nature. New York: W.H. Freeman, 1983.

Montague PR, Friedlander MJ. Morpholgenesis and territorial coverage by isolated mammalian retinal ganglion cells. J Neurosci 1989;11(5):1440-57.

Morigiwa K, Tauci M, Fukuda Y. Fractal analysis of ganglion cell dendritic branching patterns of the rat and cat retinae. Neurosci Res 1989;10:S131-40.

Murray JD. Use and abuse of fractal theory in neuroscience. J Comp Neurol 1995;361:369-71.

Neale EA, Bowers LM, Smith TG Jr. Early dendrite development in spinal cord cell cultures: a quantitative study. J Neurosci Res 1993;34:54-66.

Nonnenmacher TF, Losa GA, Weibel ER. Fractals in Biology and Medicine. Basel: Birkauser, 1994.

Panico J, Sterling P. Retinal neurons and vessels are not fractal but space filling. J Comp Neurol 1995;361:479-90.

Peitgen H-O, Jörgens H, Saupe D. Fractals for the Classroom. Part One. New York: Springer, 1992. 
Peitgen H-O, Richter P. The Beauty of Fractals. Berlin, Springer, 1986.

Porter R, Ghosh S, Lange GD, Smith TG Jr. A fractal analysis of pyramidal neurons in mammalian motor cortex. Neurosci Lett 1991;130:112-6.

$\mathrm{Pu}$ M, Berson DM, Pan T. Structure and function of the retinal ganglion cells innervating the cat's geniculate wing: An in vitro study. J Neurosci 1994;14(7):4338-58.

Ramoa AF, Campbell G, Schatz CJ. Dendritic growth and remodeling of cat retinal ganglion cells during fetal and postnatal development. J Neurosci 1988;8:4239-61.

Rasband WS, Bright DS. NIH Image: A public domain image processing program for the Macintosh. Microbeam Anal 1995; 4:137-49.

Rigaut JP. An empirical formulation relating boundary lengths to resolution in specimens showing 'non-ideally fractal' dimensions. J Microsc 1983;133(1):41-54.

Russ J. Fractal Surfaces. New York: Plenum, 1994.

Saito HA. Morphology of physiologically identified X-, Y- and W-type retinal ganglion cells of the cat. J Comp Neurol 1983;221:279-88.

Schierwagen A. Scale-invariant diffusive growth: a dissipative principle relating neuronal form to function. In: Maynard-Smith $\mathbf{J}$, Vida G, editors. Organisational Constraints on the Dynamics of Evolution. Manchester: Manchester University Press, 1990.

Schroeder M. Fractals, Chaos and Power Laws. San Francisco, CA: W.H. Freeman, 1991.

Siegel A, Reichenbach A, Hanke S, Senitz D, Brauer K, Smith TG. Comparative morphometry of Bergmann glial (Golgi epithelial) cells. Anat Embryol 1991;183:605-12.

Smith TG Jr, Behar TN. Comparative fractal analysis of cultured glia derived from optic nerve and brain demonstrate different rates of morphological differentiation. Brain Res 1994;634:181-90.

Smith TG Jr, Lange GD. Fractal studies of neuronal and glial cellular morphology. In: Iannacconne PM, Khokha M, editors. Fractal Geometry in Biological Systems. New York: CRC Press, 1995:173-88.

Smith TG Jr, Behar TN, Lange GD, Sheriff WH Jr, Neale EA. A fractal analysis of cell images. J Neurosci Methods 1989;27:17380.

Smith TG Jr, Behar TN, Lange GD, Sheriff WH Jr, Neale EA. A fractal analysis of cultured rat optic nerve glial growth and differentiation. Neuroscience 1991;41:159-66.

Smith TG Jr, Brauer K, Reichenbach A. Quantitative phylogenetic constancy of cerebellar Purkinje cell morphological complexity. J Comp Neurol 1993;331:402-6.

Smith TG Jr, Lange GD, Marks WB. Fractal methods in cellular morphology-dimensions, lacunarity and multifractals. J Neurosci Methods 1996;69:123-36.

Stanford LR. W-cells in the cat retina: Correlated morphological and physiological evidence for two distinct classes. J Neurophysiol $1987 \mathrm{a} ; 57(1): 218-244$.

Stanford LR. X-cells in the cat retina: relationships between morphology and physiology of a class of cat retinal ganglion cells. $\mathbf{J}$ Neurophysiol 1987b;58(5):940-963.

Stanford LR, Sherman MS. Structure/function relationships of retinal ganglion cells in the cat. Brain Res 1984;297:381-6.

Stell WK, Witkovsky P. Retinal structure in smooth dogfish, mustellus canis: General description and light microscopy of giant ganglion cells. J Comp Neurol 1973;148:1-32.

Stone J, Clarke R. Correlation between soma size and dendritic morphology in cat retinal ganglion cells: Evidence of further variation in the gamma cell class. J Comp Neurol 1980;192:122217.

Takayasu H. Fractals in the Physical Sciences. Manchester: Manchester University Press, 1990.

Takeda T, Ishikawa A, Ohtomo K, Kobayashi Y, Matsuoka T. Fractal dimension of the dendritic tree of cerebellar Purkinje cell during onto- and phylogenetic development. Neurosci Res 1992;13:19-31.

Viscek, T. Fractal geometry. In: Iannacconne PM, Khokha M, editors. Fractal Geometry in Biological Systems. New York: CRC Press, 1995:317-46.

Wässle H, Boycott BB. Functional architecture of the mammalian retina. Physiol Rev 1991;71(2):447-80.

Wässle H, Boycott BB, Illing RB. Morphology and mosaic of onand off-beta cells in the cat retina and some functional considerations. Proc R Soc London B Biol Sci 1981a;212:177-197.

Wässle H, Peichl L, Boycott BB. Morphology and topography of onand off-alpha cells in cat retina. Proc R Soc London B Biol Sci 1981b;212:157-175.

Wässle H, Voigt T, Patel B. Morphological and immunocytochemical identification of indoleamine-accumulating neurons in the cat retina. J Neurosci 1987;7(5):1574-87.

Welkowitz J, Ewen RB, Cohen J. Introductory Statistics for the Behavioural Sciences. New York: Academic Press, 1976.

Wingate RJT, Fitzgibbon T, Webb EI, Thompson ID. Lucifer yellow, retrograde tracers and fractal analysis characterise ferret retinal ganglion cells. J Comp Neurol 1992;323:449-74. 